\title{
The latest developments in $3 D$ visualization engine room simulators for emergency procedure training
}

\author{
R. Cwilewicz \& L. Tomczak \\ Marine Propulsion Plant Department \\ Gdynia Maritime University, Poland
}

\begin{abstract}
This paper presents the latest developments in 3D computer simulation applications, designated for the familiarization with marine machinery applied in offshore industry, especially taking into account the emergency operating procedures training. The paper describes an example of application of new 3D simulation techniques in engine room simulator based on engine room with two medium speed main engines Rolls-Royce Marine - Bergen C25:33L type, where trainees have possibilities to develop operational skills, update their know-how and refresh emergency procedures. This type of engine room is applied on offshore universal anchor handling/supply vessels. This new 3D simulation technique specially emphasizes the relation between simulation and realism of machinery operation. The paper describes also the latest development consisting in the possibility to integrate engine room simulator with bridge part. This widens the possible applications in marine simulation. The basic tasks for computer simulation in maritime education are equally described, taking into consideration the new methods and emergency procedures training.

Keywords: engine room simulators, emergency procedures.
\end{abstract}

\section{MER3D medium speed engine room simulator's description}

The application of new 3D simulation techniques in marine engineering education shall be analyzed on the examples of software type engine room simulator with medium speed main engines. The MER3D virtual reality 
simulator provides for a new approach to navigation through the different system's elements, allowing for an easy and quick access to basic engine room operation (valve opening/closing, setting position of switches, push-buttons etc.). This has been possible due the application of state-of-the art 3D visualization with zoom techniques. The latest development includes also a combination of 3D and 2D diagram presentation, which enables to follow how a certain device really functions and gives a complete picture of its structure. The presented solutions have improved considerably the level of simulator fidelity in relation to real machinery [1-3]. In consequence, it was possible to eliminate the disadvantages of the engine room simulator with typical 2D presentation consisting in a schematic and simplified presentation of machinery systems.

This simulator is designated for training students of maritime academies as well as for different types of marine vocational training centers. The simulator has universal features and may be used both for training merchant (offshore, fishery) and navy fleet crew.

MER3D engine room simulator is a perfect didactic tool as a first contact with the operation of engine room simulators. The engine room in this simulator consists only of Diesel fuel oil without steam system. MER3D engine room simulator is the new version of previous MER2 engine room simulator with 2D visualization only and is also available in hardware version with desktop type console.

The hardware console version of MER3D is presented in fig. 1 .

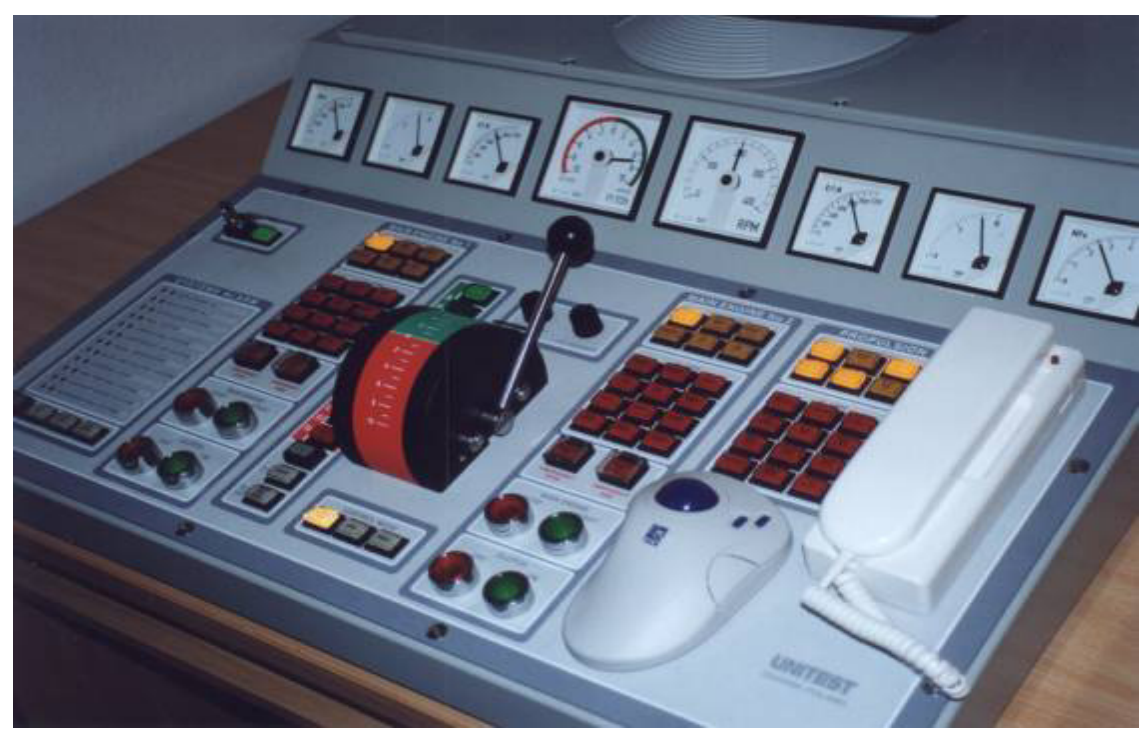

Figure 1: MER3D engine room simulator's hardware console. 
The main purpose of the MER3D simulator is the practical preparation of the trainee for engine room operation, and more particularly:

- familiarization with the basic engine room installation (compressed air system, fresh and sea water cooling system, lubricating, fuel oil, sewage, bilge water, sanitary fresh water system, sewage system, etc.)

- acknowledgment with main engine and auxiliary equipment exploitation procedures;

- $\quad$ propulsion system maneuvering ;

- fire-fighting training including $\mathrm{CO}_{2}$ and water mist system

- The software also generates the main engine room's sound.

- The simulator has been developed in compliance with:

- STCW Code: Section A-1/12 and Section B-1/12.

- ISM Code: Section 6 and Section 8.

A general view of the MER3D simulator engine room (main engines) has been presented in figs 2 and 3 .

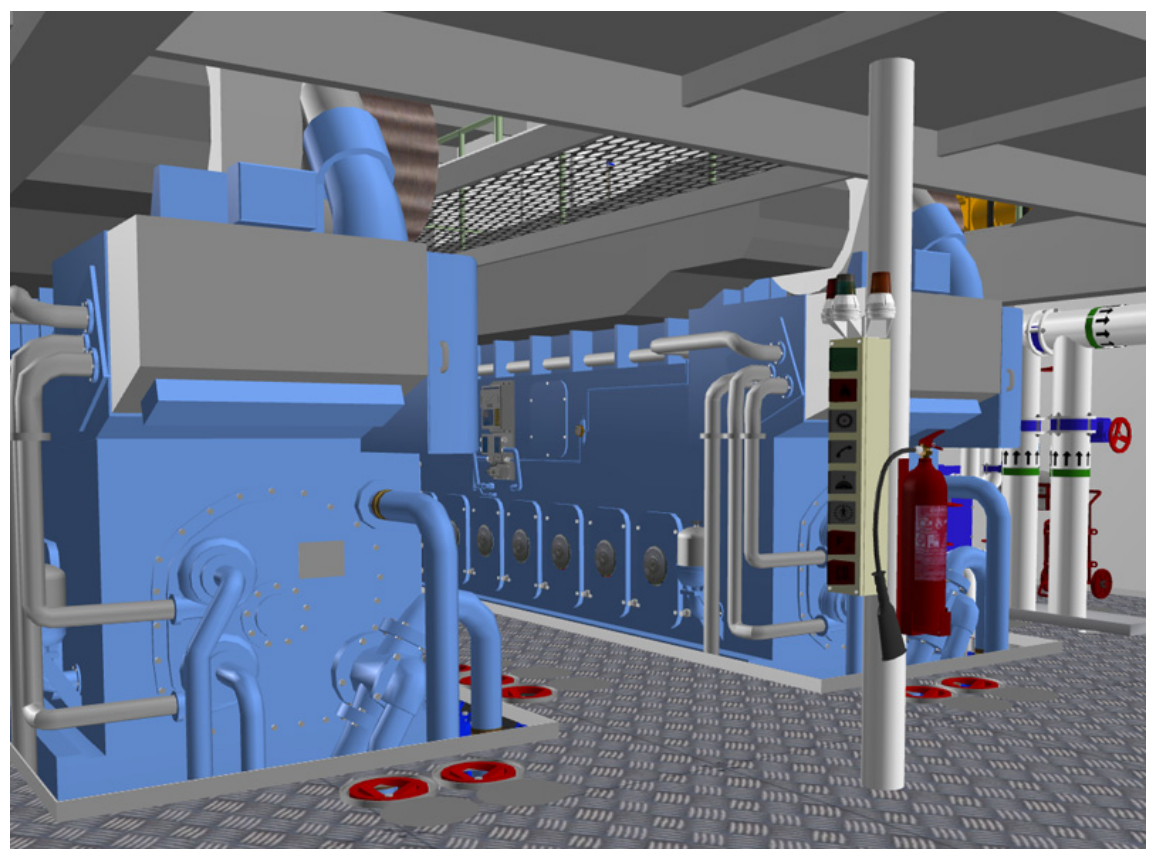

Figure 2: $\quad$ MER3D engine room simulator - main engines, general view. 


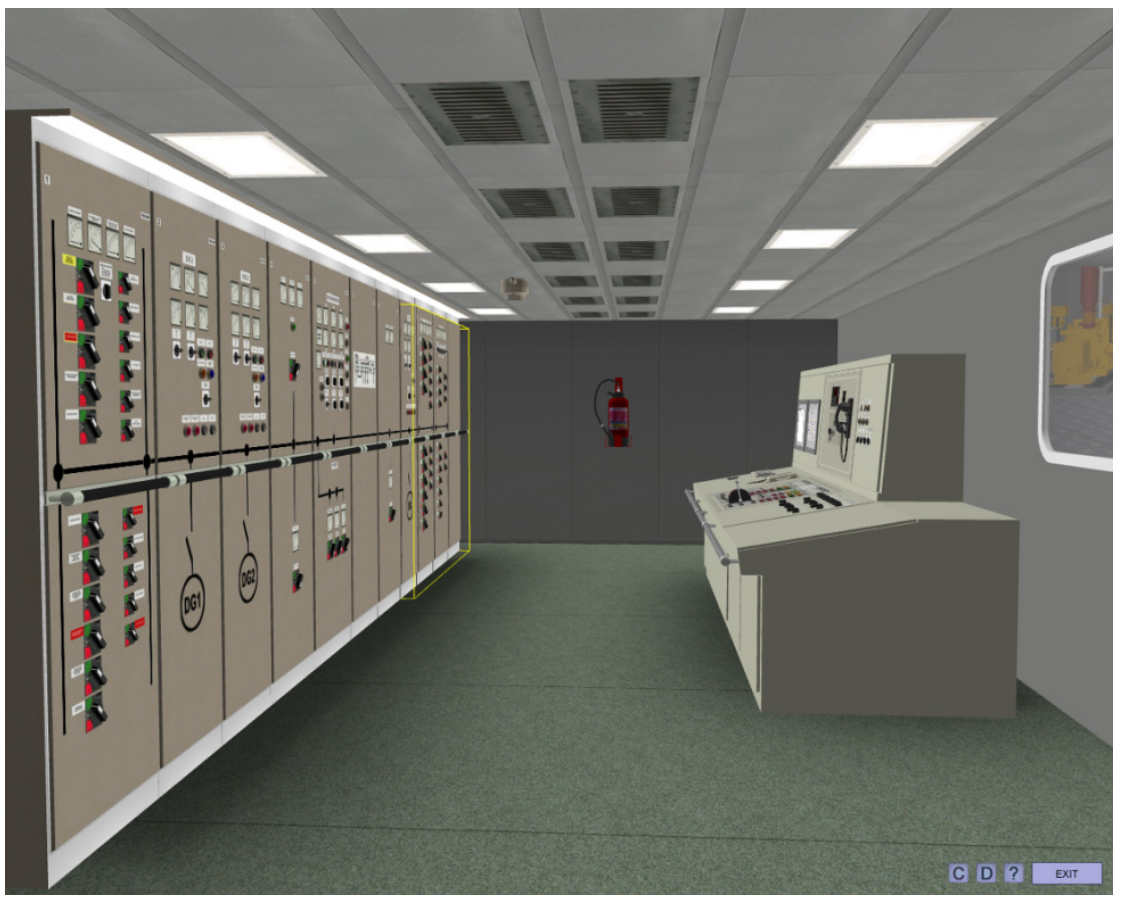

Figure 3: $\quad$ MER3D engine room simulator - control room with switchboard.

\section{Integration of 3D visualization engine room simulators with bridge part}

It this a well known fact that $80 \%$ of accidents on ships are caused by human error and half of these accidents are related to lack of communication and misunderstandings that may occur on board.

For this reason some maritime training institutions start to offer courses with application of integrated bridge and engine room simulator. Within this combined course the trainees' from deck and engine room, working together have the chance to improve their communication skills.

Not all maritime schools and vocational training centres can afford a full mission engine room simulators in a hardware version integrated with ship handling simulators. For this reason, the new engine room simulators with 3D visualization include new features related to the integration with ship's bridge. All 3D visualization simulators, like LER3D - Low Speed Engine Room simulator, MED3D Medium Speed Engine Room simulator and described in the paper MER3D Medium Speed Engine Room simulator consists of bridge part with main engine control and steering gear panel $[4,5]$. This is further developed below.

An example of the bridge part consoles is presented in figs 4 and 5 . 


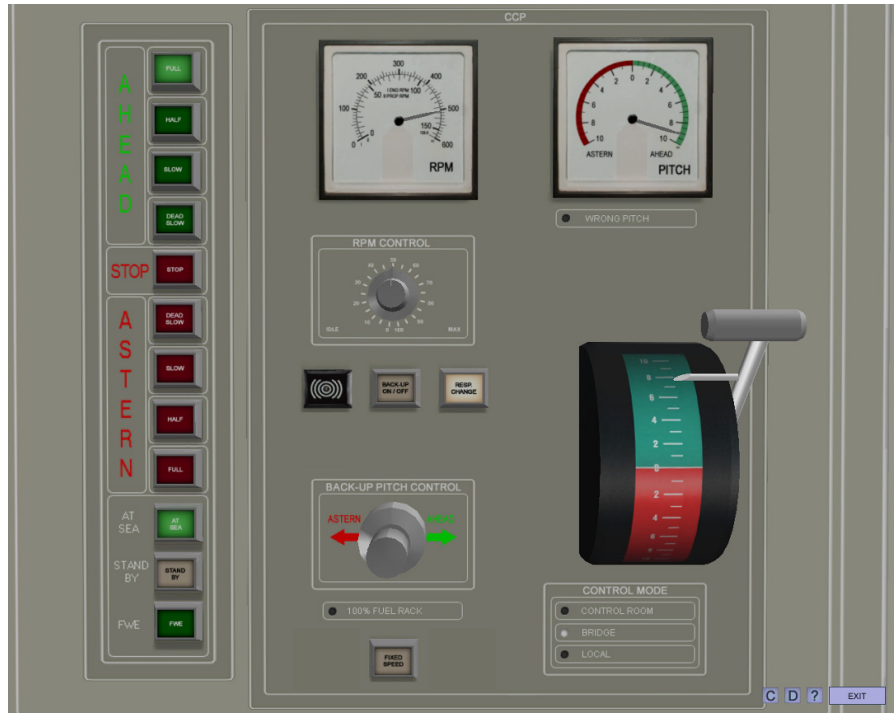

Figure 4: $\quad$ ME control panel on bridge.

In the case of integrated exercises for trainees from the bridge and engine room, the main engine and steering gear panel are separated and displayed on an additional PC connected with instructor PC.

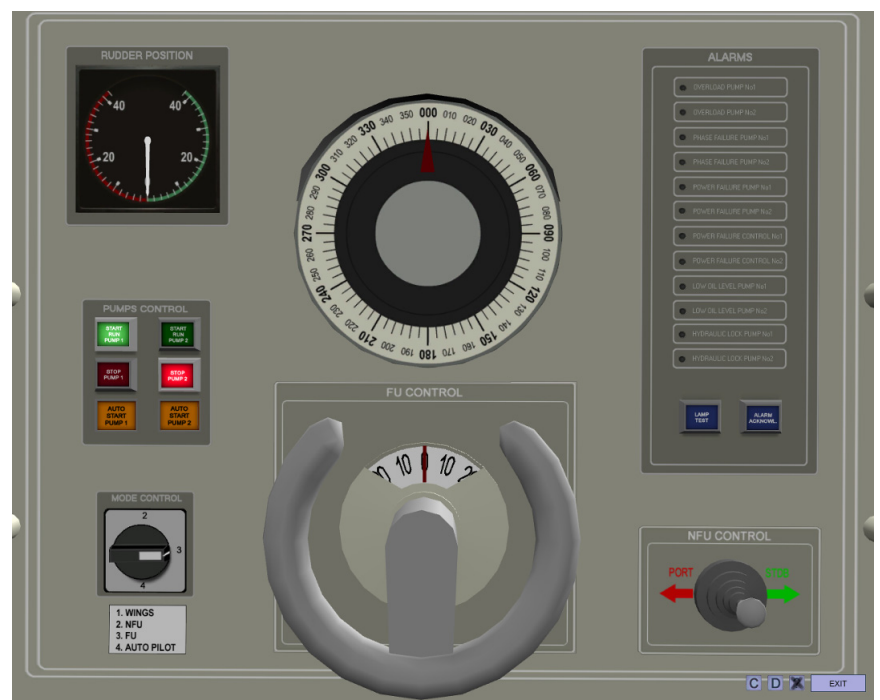

Figure 5: $\quad$ Steering gear control panel. 
The following operational modes are available:

1. Individual - non supervised by instructor trainee's activities (trainee has full access to set-ups, resources, faults etc). Instructor's programme for controlling trainee activities is not activated. Ship's bridge is not separated from engine room simulator.

2. Trainee's activities supervised by instructor (only instructor has access to set-ups, resources, faults etc). Instructor's programme for controlling trainee's activities is running. The ship's bridge is not separated and the instructor's and trainee's PCs are connected as network. Trainees from the deck do not participate in training but only the trainees from engine room.

3. Trainees' activities supervised by instructor (only instructor has access to set-ups, resources, faults etc). Instructor's programme for controlling trainees' activities is working. The ship's bridge is separated from engine room and the PC's of the instructor, the trainees for both engine room and bridge are connected as network. In this case trainees from deck and engine room operate together.

With 3D visualisation application of the engine room and bridge, the training becomes very realistic and more effective.

The main problem in integrated courses is preparation of proper exercise's scenarios.

Following scenarios are proposed:

1. Bridge-engine control room communication during main engine preparation for starting including changing command from engine control room to bridge.

2. Failure of Main Engine (ME) control from the bridge - change to the Engine Control Room (ECR).

3. Failure of ME control from bridge and ECR - change to ME local control. Fig. 6 shows the example of local control panel applied on LER3D engine room simulator.

4. Failure of follow-up steering gear operation - change to non follow-up control.

5. Failure of follow-up and non follow-up steering gear operation - change to engine room local control. Engine room steering gear (rotary vane type) local control panel is presented in figs 7 and 8 .

6. Black-up in engine room - emergency generator's batteries operational

7. Black-up in engine room - emergency generator's batteries not operational.

8. Fire in ME scavenge box of in one selected ME cylinder.

9. Fire in engine room $-\mathrm{CO}_{2}$ pilot bottles operational (fig. 9).

10. Fire in engine room $-\mathrm{CO}_{2}$ pilot bottles not operational. 


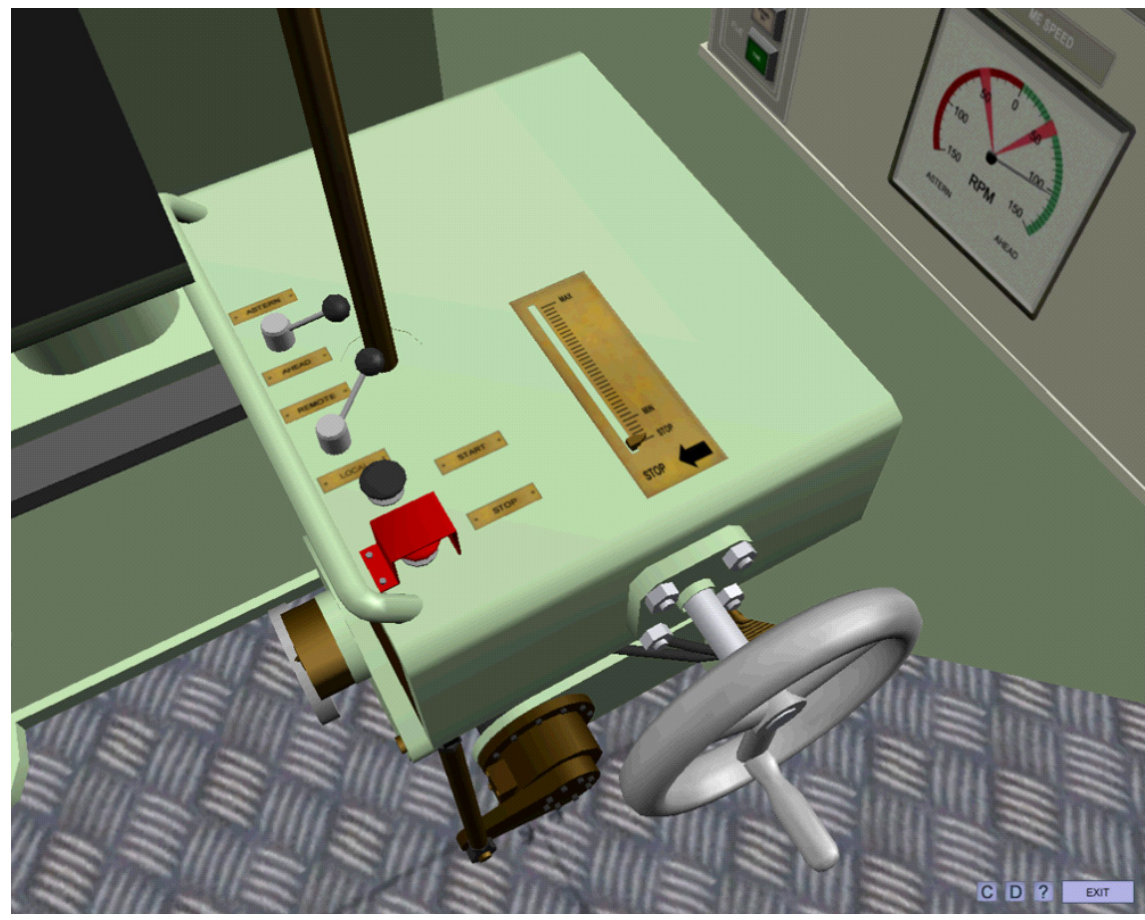

Figure 6: $\quad$ ME local control panel.

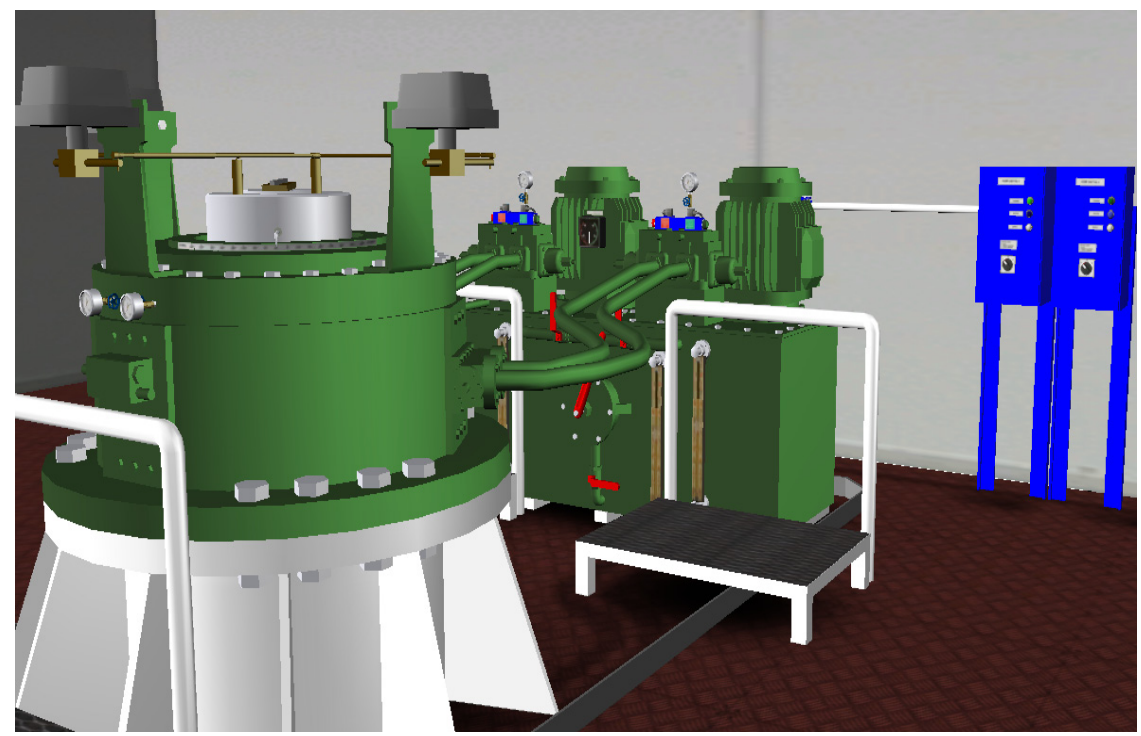

Figure 7: $\quad$ Steering gear general view. 


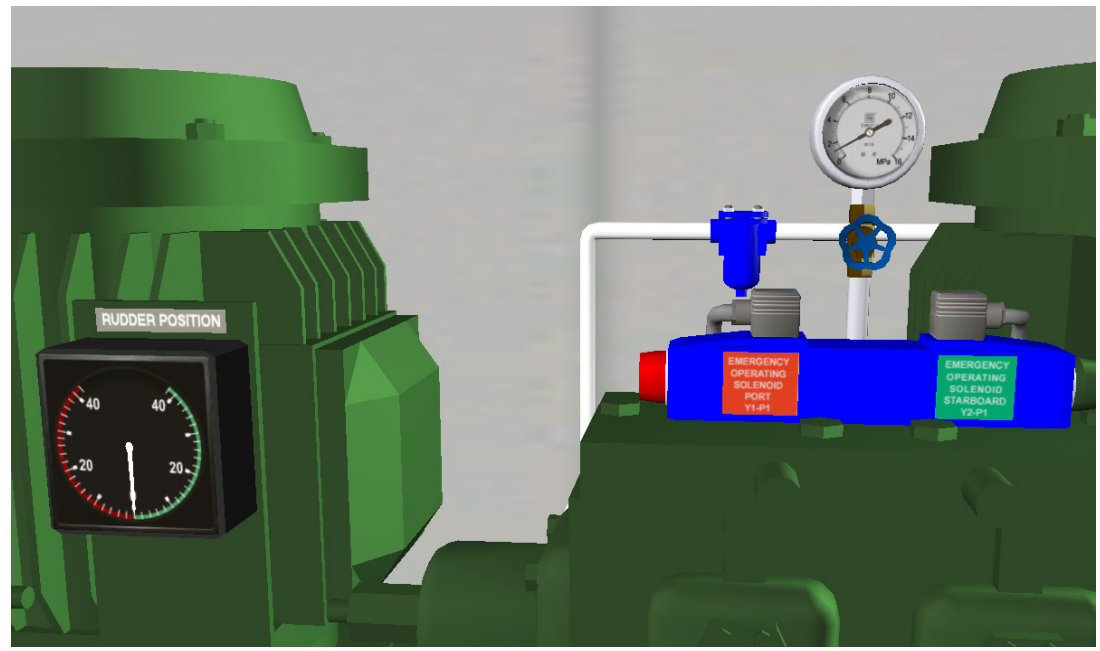

Figure 8: Directional valve push-buttons for local emergency steering gear control.

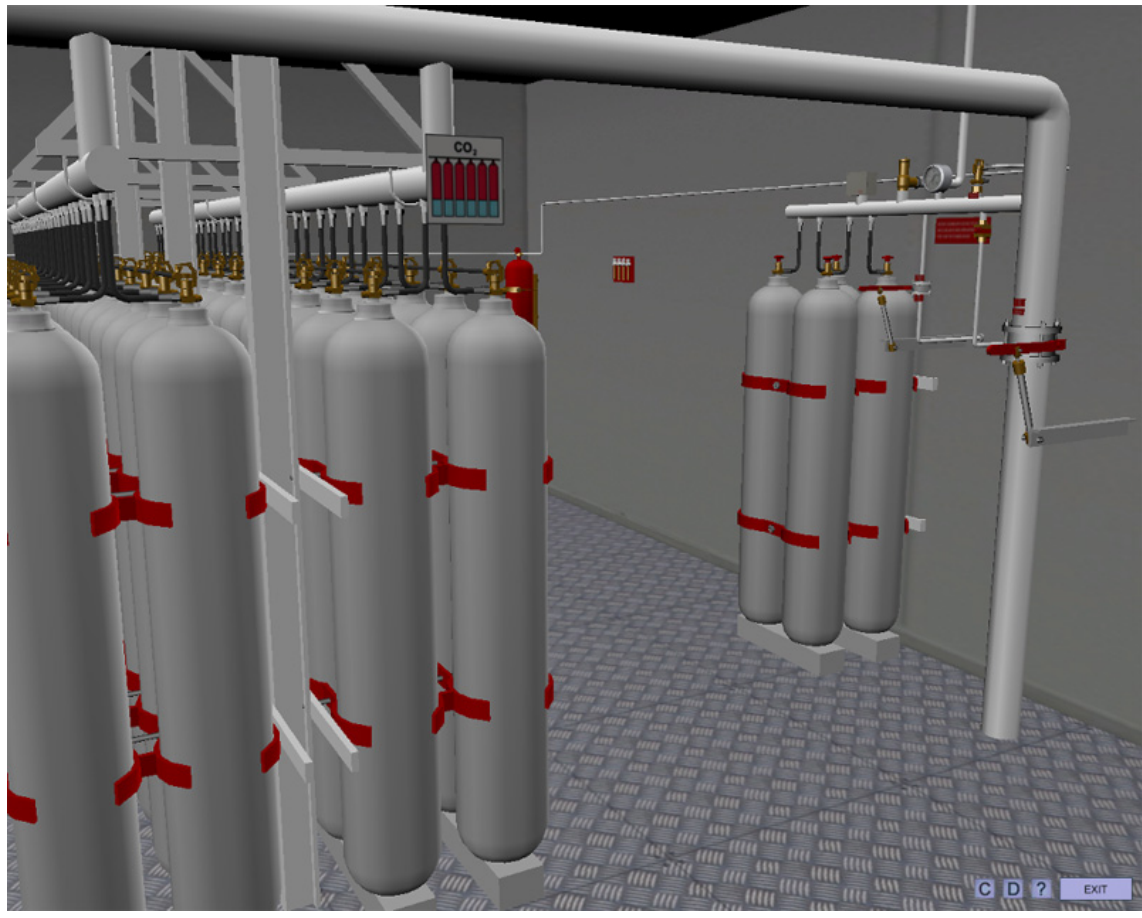

Figure 9: $\quad$ Fire-fighting system - general view. 


\section{Conclusions}

The application of engine room simulators with 3D visualization in maritime education leads to a better understanding of the marine machinery and results also in increased emergency preparedness and in consequence, leads to hazard mitigation and reduces the risk of human error in the operation and maintenance of marine equipment.

As it results from the above, the latest developments in simulation techniques, including $3 \mathrm{D}$ presentation enhances the above mentioned benefits, as it brings the engine room simulators closer to reality. In consequence, the gap between operating marine machinery in simulation conditions and in real life is decreased.

As it has been mentioned above, proper navigation through the 3D simulator's elements is the key point in order to achieve the didactic purposes. The new concepts of view selection, zooming features of elements and operation by cursor and mouse clicking, as applied in MER3D medium speed engine room simulator, described in this paper, appears to be very effective and easily adaptable by trainees in practice.

Due to the specificity of operating marine equipment in real life conditions, the didactic goals in marine education are directly linked with achieving preparedness for emergency situations. Such preparedness may only be achieved if the trainee is familiar with both the equipment and its operating modes, including emergency situations.

In the near future, this type of 3D solutions should be applied more and more often in engine room simulators design. The presented simulators are related to marine machinery, but the concept of composition and navigation through the system's elements can be easily applied for the purposes of any type of technical equipment.

Integration of engine room and bridge part constitutes and important improvement leading to better communication skills. The new engine room simulator software version with $3 \mathrm{D}$ visualization with possibility to separate the bridge part offers an innovative and at the same time inexpensive didactic tool, which can be easily applied in different marine training institutions since it does not require the same budget expenses as a hardware version. However, to be effective this new feature requires the preparation of proper exercises' scenarios taking into consideration an active participation of trainees both from the deck and from the engine room.

\section{References}

[1] Tomczak L., Practical aspects of 3D graphical applications in marine Engineering Education, Global Journal of Engineering Education, 9(2), 2005.

[2] Tomczak L., Application of 3D visualization in marine engine room simulators, Proc. $7^{\text {th }}$ International Conference on Engine Room Simulators (ICERS7), Portoroz, Slovenia, 2005. 
[3] Cwilewicz R., Tomczak L., Application of 3D computer simulation for marine engineers as a hazard prevention tool, Proc $5^{\text {th }}$ International Conference on Computer Simulation In Risk Analysis and Hazard Mitigation - Risk Analysis V,WIT PRESS Southampton, Boston, 2006.

[4] Tomczak L., The latest developments of $3 \mathrm{D}$ visualization in marine engine room simulators, Proc. International Conference on Engine Room Simulators (ICERS8), Manila, Philippines, 2007.

[5] Cwilewicz R., Tomczak L., Improvement of ship operations' safety as a result of the application of virtual reality engine room simulators Proc. $6^{\text {th }}$ International Conference on Computer Simulation In Risk Analysis and Hazard Mitigation - Risk Analysis VI, WIT PRESS Southampton, Boston, 2008.

[6] Tomczak L., The new generation of engine room simulators with application of 3D visualization, Journal of Polish CIMAC, Gdańsk, Poland Vol.3 No. 2, 2008. 